\section{AB308 IS AN ANTI-TIGIT ANTIBODY THAT ENHANCES IMMUNE ACTIVATION AND ANTI-TUMOR IMMUNITY ALONE AND IN COMBINATION WITH OTHER I-O THERAPEUTIC AGENTS}

Dana Piovesan*, Alejandra Lopez, Patrick Schweickert, Ferdie Soriano, Soonweng Cho, Ada Chen, Hema Singh, Xiaoning Zhao, Stephen Young, Nigel Walker, Matthew Walters, Kelsey Sivick Gauthier. Arcus Biosciences, Hayward, CA, USA

Background TIGIT (T-cell immunoreceptor with Ig and ITIM domains) is an inhibitory receptor expressed on natural killer (NK) cells, CD8 $<$ sup $>+</$ sup $>\mathrm{T}$ cells, $\mathrm{CD} 4<$ sup $>+</$ sup $>\mathrm{T}$ cells and regulatory $\mathrm{T}$ cells $(\mathrm{T}<$ sub $>$ regs $<$ /sub $>$ ). On the surface of these cells, TIGIT competes with another receptor, CD226, for shared receptor ligands (mainly CD155) that are expressed by cancer and antigen-presenting cells. Binding of CD155 to TIGIT results in immune suppression through multiple mechanisms. When TIGIT is blocked, binding of CD155 to $\mathrm{CD} 226$ promotes immune activation and anti-tumor immunity. We describe the preclinical characterization of AB308, a humanized wild-type IgG1 anti-TIGIT antibody that is currently undergoing clinical evaluation.

Methods Binding of AB308 to TIGIT and inhibition of the TIGIT/CD155 interaction were evaluated $<\mathrm{i}>$ in vitro $</ \mathrm{i}>$. Functional assays were used to evaluate the immunomodulatory activity of $\mathrm{AB} 308$ alone or in combination with zimberelimab (anti-PD-1) or etrumadenant (a small molecule $\mathrm{A}<\mathrm{sub}$ $>2 \mathrm{a}</$ sub $>\mathrm{A}<$ sub $>2 \mathrm{~b}</$ sub $>$ adenosine receptor antagonist). Surrogate Fc-silent and Fc-enabled antibodies that recognize mouse TIGIT or PD-1 were leveraged to interrogate TIGIT biology in syngeneic mouse tumor models.

Results Human tumor-infiltrating lymphocytes from a variety of cancer types expressed appreciable levels of TIGIT on relevant immune populations, including tumor reactive $\mathrm{CD} 39<$ sup $>+</$ sup $>$ CD103 $<$ sup $>+</$ sup $>$ CD $8<$ sup $>+<1$ sup $>\mathrm{T}$ cells and $\mathrm{T}<$ sub $>$ regs $</$ sub $>$. AB308 has a high binding affinity for human TIGIT, potently blocks the TIGIT$\mathrm{CD} 155$ interaction, and induces $\mathrm{F} c \gamma$ receptor $(\mathrm{Fc} \gamma \mathrm{R})$-mediated signaling. In line with FcyRIII binding, AB308 also demonstrated the ability to induce NK cell-driven antibody-dependent cell-mediated cytotoxicity against TIGIT-expressing target cells. AB308 significantly increased IL-2 secretion by peripheral blood mononuclear cells activated with superantigen A, an activity that was further enhanced with zimberelimab. Blocking TIGIT with AB308 potently activated CD226 signaling in Jurkat $\mathrm{T}$ cells co-cultured with CD155-expressing cells, and combination of $\mathrm{AB} 308$ with etrumadenant in this system abrogated adenosine-mediated $\mathrm{T}$ cell suppression that occurred even in the presence of checkpoint inhibition. In mice, while combining $\mathrm{Fc}$-silent or Fc-enabled anti-mouse TIGIT antibody with anti-PD-1 resulted in greater tumor growth inhibition than with anti-PD-1 alone, the activity of Fc-enabled antiTIGIT was associated with intratumoral $\mathrm{T}<$ sub $>$ regs $</$ sub $>$ depletion.

Conclusions AB308 is a potent and highly effective anti-TIGIT antibody. Concurrent blockade of multiple immune checkpoints has the potential to confer effective and durable responses in the treatment of cancer. The data presented here support the clinical use of $\mathrm{AB} 308$ and provides a rationale for combination with zimberelimab and adenosine pathway blocking agents such as etrumadenant and CD73 small molecule inhibitor, AB680.

Ethics Approval Animal experiments were performed at Arcus Biosciences, Inc. in accordance with federal, state and
Institutional guidelines and were approved by Arcus' Institutional Animal Care and Use Committee.

http://dx.doi.org/10.1136/jitc-2021-SITC2021.258 